\title{
Gradhiva
}

GRADHIV

Revue d'anthropologie et d'histoire des arts

25 | 2017

Gottfried Semper, habiter la couleur

\section{Valérie Da Costa, Pino Pascali : retour à la}

Méditerranée

Dijon, Les presses du réel, 2015

\section{Laura lamurri}

\section{OpenEdition}

\section{Journals}

Édition électronique

URL : http://journals.openedition.org/gradhiva/3343

DOI : $10.4000 /$ gradhiva.3343

ISSN : 1760-849X

\section{Éditeur}

Musée du quai Branly Jacques Chirac

Édition imprimée

Date de publication : 31 mai 2017

Pagination : 259-261

ISBN : 978-2-35744-095-1

ISSN : 0764-8928

\section{Référence électronique}

Laura lamurri, « Valérie Da Costa, Pino Pascali : retour à la Méditerranée », Gradhiva [En ligne], 25 | 2017, mis en ligne le 31 mai 2017, consulté le 24 septembre 2020. URL : http://journals.openedition.org/ gradhiva/3343 ; DOI : https://doi.org/10.4000/gradhiva.3343

Ce document a été généré automatiquement le 24 septembre 2020.

(c) musée du quai Branly 


\section{Valérie Da Costa, Pino Pascali : retour}

\section{à la Méditerranée}

Dijon, Les presses du réel, 2015

Laura lamurri

\section{RÉFÉRENCE}

Valérie Da Costa, Pino Pascali : retour à la Méditerranée. Dijon, Les presses du réel, 2015, $308 \mathrm{p}$.

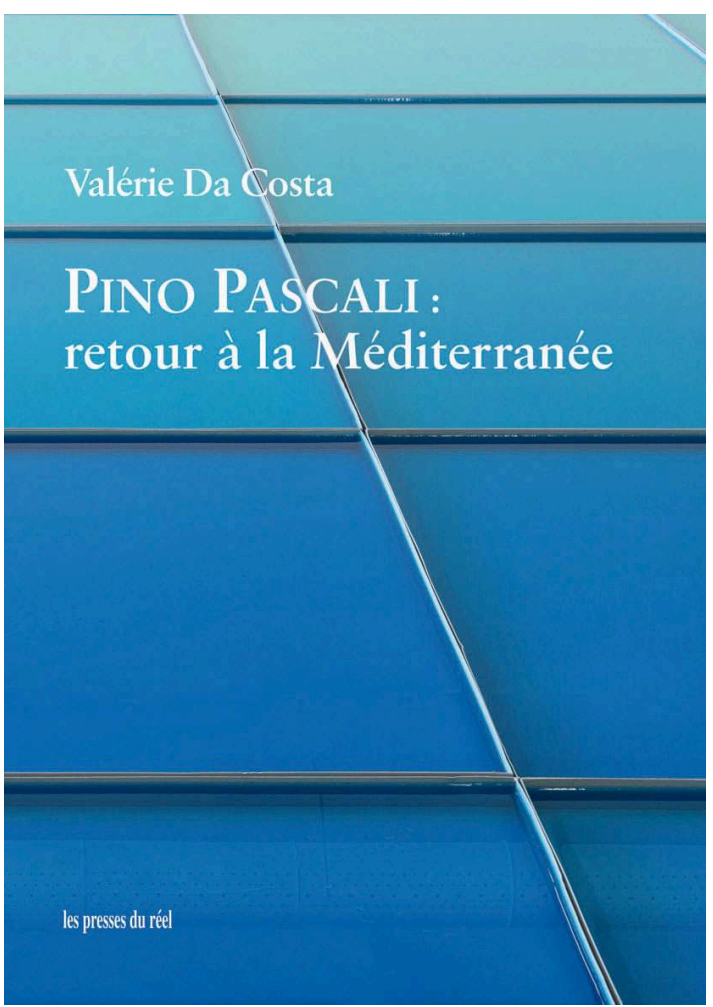


1 Pino Pascali a été l'un des grands artistes de l'après-guerre. Il a traversé comme un météore le ciel de l'art italien des années 1960 : depuis ses débuts sur la scène romaine avec sa première exposition personnelle en janvier 1965 jusqu'à sa mort après un accident de la route le 11 septembre 1968 n'ont passé que trois ans et demi. Années extraordinairement riches d'œuvres et d'inventions plastiques, d'expérimentations sur les matériaux et de réflexions sur la sculpture, de subversions de l'espace et de la conception de l'exposition contemporaine. Avec son œuvre plastique, il a réinventé tout un monde, naturel et artificiel, à l'apparence joyeuse et ludique.

2 Habituellement associé au groupe de l'arte povera, Pascali reste assez peu connu hors de l'Italie. La monographie que lui consacre Valérie Da Costa est la première en langue française et, à divers égards, fait preuve d'originalité comparée aux nombreuses études italiennes. L'ouvrage est issu d'un travail de recherche considérable, une véritable enquête dans nombre d'archives publiques et privées, et notamment dans celles de la Fondazione Museo Pino Pascali à Polignano a Mare, dans les Pouilles, où l'artiste est né en 1935. Le discours critique et historique, extrêmement soigné et documenté de bout en bout, est soutenu par la qualité et la quantité des images (reproductions d'œuvres et de documents, dessins et esquisses, notes manuscrites et lettres, photographies d'atelier, d'accrochages, de chroniques mondaines et artistiques) qui éclairent parfois le texte de manière exemplaire.

3 Le livre s'ouvre à l'été 1968, le dernier de la brève existence de Pascali : à la fois été de sa consécration avec une salle à la Biennale de Venise et de la contestation de cette institution de la part des étudiants et d'un certain nombre d'artistes. Après cette introduction, qui nous montre la scène de la culture italienne de l'époque et ses principaux acteurs, Da Costa engage une relecture de l'œuvre de Pascali, comme elle l'écrit elle-même, « en fonction des thèmes » (p. 31): mutations de la sculpture, de sa temporalité et de sa spatialité ; construction d'une identité d'artiste et de sa mise en scène; attachement à la Méditerranée qui donne le titre à l'ouvrage et en constitue le contexte de référence; enfin, l'intérêt de Pascali pour l'anthropologie, et tout particulièrement pour cette anthropologie qui faisait du sud de l'Italie l'objet d'études importantes, notamment de la part d'Ernesto De Martino.

4 La structure thématique du livre n'exclut pas une démarche chronologique au sein de chaque section regroupant les œuvres suivant les différents axes conceptuels. Cette double entrée permet à Da Costa de s'arrêter également sur les années de formation de Pascali à l'Accademia di Belle Arti di Roma où il a été le compagnon d'études de Jannis Kounellis et l'élève en scénographie de Toti Scialoja. Les dessins des années 1958-1959 montrent déjà l'usage des caractères d'imprimerie à stencil - que l'on retrouvera encore estampillés sur les sculptures exposées en 1965 à la Galleria La Tartaruga lors de sa première exposition personnelle - ainsi qu'une certaine désinvolture dans la mise en pages, qui devient la marque d'une liberté de création effrénée, également expérimentée durant ses années de collaboration avec l'agence de publicité de Sandro Lodolo (1958-1967). Bien que la production publicitaire de Pascali et son travail pour la RAI (la télévision publique italienne) soient désormais bien connus, c'est l'un des mérites de l'auteure d'avoir souligné que l'artiste cesse ces collaborations «alimentaires » seulement en 1967, moment où le soutien du galeriste Alexandre Iolas lui permet enfin de se consacrer pleinement à la sculpture.

5 La sculpture n'aura donc pas été, durant une grande partie de sa vie, l'activité exclusive de Pascali. Son goût pour ce que Da Costa appelle « une certaine technicité artisanale » 
(p. 138), la maîtrise d'un savoir-faire qu'il revendique ouvertement, caractérise toute son œuvre, qu'il s'agisse de l'invention quotidienne d'un langage publicitaire pour la télévision ou de la révolution permanente de sa sculpture. Les photographies prises par Claudio Abate dans l'atelier de l'artiste témoignent, dit l'auteure, de "l'organisation méthodique de tous ses outils, digne de l'atelier d'un grand bricoleur » (p. 138) et de sa passion pour la transformation de la matière. Quand en 1965 il travaille à sa série des Armi (Armes), sa technique renoue avec l'un des procédés éprouvés de l'avant-garde : l'assemblage, une technique qui mêle goût de la recherche, esprit de collecte et montage d'objets variés. En l'occurrence, chez Pascali, celui de pièces métalliques devenues inutiles, de restes et fragments d'engins ou de carrosseries. Si ce même procédé ne pouvait manquer de rappeler aux Romains l'usage qu'en avait fait le sculpteur Ettore Colla (1896-1968), contrairement à ce dernier qui avait forcé les éléments métalliques à l'intérieur de compositions abstraites au rythme quasiment classique, Pascali utilisait la méthode de l'assemblage pour réaliser des constructions qui devaient passer pour de vrais objets : peintes uniformément en vert militaire, ses armes - des jouets géants, inutiles, inoffensifs - donnaient l'impression visuelle de vraies armes.

6 Les ambiguïtés de ces œuvres au regard de l'idée de la sculpture sont évidentes : face à ces grands «objets» déconcertants, les réactions ne sont pas toujours enthousiastes. Pourtant, grâce à la médiation de Michelangelo Pistoletto, les Armes sont vite exposées à Turin: Da Costa reproduit et traduit dans son livre les notes - restées jusque-là inédites - rédigées par Pascali en vue de l'exposition et la correspondance avec le galeriste qui allait montrer ses nouvelles œuvres. Gian Enzo Sperone, jeune marchand attentif aux langages contemporains et aux récentes saillies "pop ", avait inauguré sa direction de la galerie Il Punto avec la première exposition italienne de Roy Lichtenstein; en 1964, un nouvel espace à son nom - la Galleria Sperone - avait accueilli les œuvres de Christo, Robert Rauschenberg, James Rosenquist; en 1965 une nouvelle première italienne, celle d'Andy Warhol, mais aussi de Jim Dine, Arman et Martial Raysse. Les Armes sont donc présentées à un public turinois bien informé, et ne manquent pas de susciter l'intérêt des confrères artistes et de quelques critiques avisés.

L'ambivalence de la sculpture - cette oscillation continue entre la rhétorique de l'image et la nomination des objets, entre l'évocation de la guerre et le caractère évidemment et absolument inoffensif - évolue rapidement et se fait de plus en plus audacieuse. La démarche de Pascali le conduit à explorer de nouvelles pistes, à élargir le champ de la sculpture aux grandes formes blanches qu'il appelle les «fausses sculptures » : fausses parce qu'elles sont réalisées avec les matériaux de la peinture - toile tendue sur des cintres en bois - et que la couleur blanche leur donne une allure moins statuaire que fantomatique. Il les présente en 1966 chez Fabio Sargentini, son nouveau galeriste à Rome, en deux étapes successives ${ }^{1}$ : la première, inaugurée à la fin du mois d'octobre, est consacrée à la série des animaux avec des grandes formes aux titres bizarres tels la Decapitazione del rinoceronte, la Decapitazione delle giraffe, i Dinosauri, etc. (Décapitation du rhinocéros, Décapitation des girafes, Dinosaures, etc.) ; la seconde exposition, en novembre, est entièrement conçue autour de la mer, avec deux grandes Balene (Baleines) accrochées au mur, la Barca che affonda (Bateau qui coule), les éléments de la Scogliera (Falaise) et enfin Il mare ( $\mathrm{La} \mathrm{Mer)} \mathrm{:} \mathrm{une} \mathrm{"sculpture} \mathrm{"} \mathrm{composée} \mathrm{de} \mathrm{vingt-quatre} \mathrm{pièces}$ carrées étalées au sol figurant les vagues qui occupent presque la totalité de la salle, obligeant le public à se tenir sur le pourtour de l'installation. Bien que les références au futurisme et notamment à Giacomo Balla (qui avait vécu à Rome jusqu'à sa mort en 
1958) soient nombreuses dans l'œuvre de Pascali, le spectateur n'est pas placé «au centre du tableau ", comme on pouvait le lire dans le manifeste de la peinture futuriste : au contraire, le public reste sur le seuil de la galerie, car le protagoniste absolu de l'espace est l'artiste même. Lui seul, souligne Da Costa, tient le rôle principal dans la mise en scène de soi devant l'appareil photo et dans l'invention d'une qualité théâtrale de l'espace dont il a au même moment fait l'expérience en suivant les activités du Living Theatre à Rome.

Les «fausses sculptures" marquent à plusieurs niveaux un passage important: la réinvention de la mer est, entre autres, une puissante évocation de la Méditerranée de l'enfance de Pascali. L'artiste en parle avec Carla Lonzi, une critique d'art exceptionnellement proche des artistes et attentive à leurs idées, pensées, projets. Dans une conversation parue en 1967 dans la revue Marcatrè puis rééditée dans le livre Autoritratto, Pascali rappelle sa passion pour la mer des Pouilles où, enfant, il a passé toutes ses vacances d'été. Dans l'urgence qui paraît caractériser son travail, dans l'invention presque quotidienne de combinaisons inédites de nouveaux matériaux, Pascali annonce à Lonzi : «Les prochaines choses que je veux faire sont des choses d'eau ${ }^{2} »($ p. 167) ; quand la revue est mise en vente, il a déjà réalisé et exposé les $9 \mathrm{mq} d i$ pozzanghere ( $9 \mathrm{~m}^{2}$ de flaques) chez Sargentini, et les $32 \mathrm{mq}$ di mare circa $\left(32 \mathrm{~m}^{2} \mathrm{de}\right.$ mer environ) à Foligno, dans l'exposition «Lo spazio dell'immagine », la première, en Italie, dédiée aux installations. Comme le souligne Da Costa, l'œuvre de Pascali évoque «l'idée d'une totale occupation de l'espace par l'eau » (p.177) et oblige en même temps à mesurer l'écart entre l'étendue de la mer et son découpage en morceaux, en bassins métalliques encore une fois étalés au sol; la couleur de l'eau, graduée du bleu pâle au bleu foncé, suggère la profondeur - inexistante - de la mer.

9 Cette récréation de la nature avec des matériaux artificiels n'est pas alors une prérogative de Pascali, qui - pour nous en tenir à l'Italie - partage cette idée d'une réinvention du monde avec d'autres artistes, tels Piero Gilardi ou Gino Marotta. Mais à l'inverse de ces derniers, Pascali ne se limite pas à utiliser des matériaux artificiels plus ou moins issus de l'industrie moderne, il y mêle des éléments naturels : la mer est donc véritablement faite d'eau tout comme le $m^{3}$ de terre présente une surface extérieure recouverte de terre. On a toujours l'impression, avec Pascali, d'une série de doubles mouvements, entre culture et nature, entre spontanéité apparente et stratégies de mise en scène, entre la fréquentation de l'avant-garde italienne, voire internationale, et le retour constant à la province, à la culture paysanne qu'il revendique ostensiblement quand il utilise le dialecte dans le catalogue d'une exposition ou récite une comptine de son enfance en ouverture de son entretien avec Marisa Volpi ${ }^{3}$.

Cette fierté, cette origine méridionale exhibée ont retenu l'attention de Da Costa, qui pour la première fois a proposé une lecture des dernières œuvres de Pascali, et notamment de la Vedova blu (Veuve bleue), à la lumière des textes d'Ernesto De Martino. Historien des religions et ethnologue, De Martino avait publié au début des années 1960 son étude sur le phénomène du tarentisme, rite de la religion populaire répandu dans le Salento (Pouilles méridionales) que l'auteur définissait comme "un esorcismo coreutico-musicale ${ }^{4}$ " ( un exorcisme chorégrapho-musical»). L'hypothèse de Da Costa est passionnante et convaincante : si Pascali n'a pas lu La Terra del rimorso, il pourrait bien avoir vu le film documentaire $L a$ Taranta que Gianfranco Minguzzi avait réalisé en 1962 à partir de l'étude de De Martino. La Vedova blu constitue per se un indice qui va dans ce sens, par sa référence à l'araignée (la veuve noire, mais il en existe une variété 
bleuâtre) dont, dans la tradition populaire du Salento, la morsure déclenche la manifestation du tarentisme. Une photo de Claudio Abate, encore une fois véritable complice de l'artiste dans la construction de son image, nous montre Pascali à Rome, au pied de sa grande sculpture, en une sorte de roulade qui paraît imiter de manière ludique les contorsions des tarentules sur le sol.

Tandis que la liste des matériaux utilisés par l'artiste s'allonge (à la fourrure synthétique de la Vedova blu s'ajoutent les brosses à nettoyer des Bachi da setola, la laine d'acier de la série des ponts et des arcs et des Penne di Esopo (Plumes d'Ésope), aujourd'hui dans les collections du musée national d'Art moderne à Paris, de nouvelles œuvres renouent avec le caractère rural du Sud de l'Italie : les Campi arati (Champs labourés), les Canali d'irrigazione (Canaux d'irrigation) et les Strumenti agricoli (Outils agricoles), tous réalisés en 1968, qui témoignent de la conscience aiguë des relations entre centre et périphérie, des transformations du territoire et des activités humaines, également comme Da Costa le rappelle - au cœur des réflexions, voire des préoccupations, de Pier Paolo Pasolini. Au moment où sa carrière prend une tournure internationale, avec la multiplication des expositions à l'étranger et l'invitation à la Biennale de Venise, Pascali vise à créer un équilibre difficile entre la nouvelle dimension cosmopolite de son expérience d'artiste et la réinvention d'un monde paysan et rural, source inépuisable d'une culture jugée authentique parce que provinciale, traditionnelle et minoritaire.

En furetant dans ce qui reste de la bibliothèque de Pascali, Da Costa a certainement contribué à une nouvelle compréhension de l'artiste, bien plus complexe que celle donnée par ses entretiens, où domine une tendance presque compulsive à la divagation. Écarter les fausses pistes disséminées par l'artiste (et par ses amis) n'était pas un exercice banal, mais il est réussi : en puisant à différentes typologies de sources écrites et visuelles, ce livre issu d'un remarquable travail de recherche atteint des résultats inédits en France comme en Italie, grâce à la validité de son hypothèse critique et à la qualité de la réflexion qui accompagne l'exégèse des sources. Ouvrage de référence, Pino Pascali : retour à la Méditerranée soulève bien des questions et ouvre des nouvelles pistes de réflexion méthodologique sur l'art italien des années soixante.

\section{NOTES}

1. Voir Pino Pascali : nuove sculture, cat. expo., Rome, Galleria L'Attico, 1966.

2. Voir la déclaration de l'artiste dans «Pino Pascali e Carla Lonzi., Discorsi », Marcatrè 30-33, 1967 : 239-245 ; in Carla Lonzi, Lara Conte, Laura Iamurri, Vanessa Martini (dir.), Scritti sull'arte. Milan, et al. edizioni, 2012 : 525-537 (déclaration de Pascali : 535). Voir Carla Lonzi, Autoritratto. Bari, De Donato, 1969 : 385/Milan, et al. edizioni, $2010: 293$; trad. de l'italien par Marie-Ange Maire-Vigueur : Autoportrait. Zurich/Paris, JRP Ringier/ La Maison rouge, 2012 : 207.

3. Voir la réponse de Pascali aux questions posées par Marisa Volpi dans le cadre d'une enquête sur les techniques et les matériaux dans l'art italien : Carla Lonzi, Tommaso Trini et Marisa Volpi, «Tecniche e materiali », Marcatrè 37-40, 1968 : 66-85 (entretien reproduit in Carla Lonzi, Scritti sull'arte, op. cit. : 560-610; réponses de Pascali : 577-578). 
4. Ernesto De Martino, La terra del rimorso: contributo a una storia religiosa del Sud. Milan, Il Saggiatore, 1961 ; trad. de l'italien par Claude Poncet : La Terre du remords. Paris, Gallimard, 1966/ Le Plessis-Robinson, Institut Synthélabo, 1999.

\section{AUTEURS}

\section{LAURA IAMURRI}

lauraiamurri@gmail.com 